\title{
Características da sucção nutritiva na liberação da via oral em recém- nascidos pré-termo de diferentes idades gestacionais
}

\author{
Characteristics of nutritive sucking in the release for oral feeding in \\ preterm newborns of different gestational ages
}

\author{
Raquel Coube de Carvalho Yamamoto ${ }^{1}$, Márcia Keske-Soares², Ângela Regina Maciel Weinmann ${ }^{3}$
}

\begin{abstract}
RESUMO
Objetivo: Analisar as características da sucção nutritiva na liberação da via oral em recém-nascidos pré-termo (RNPT) de diferentes idades gestacionais. Métodos: A amostra constou de 32 RNPT, avaliados no momento da liberação para alimentação por via oral, dividida em dois grupos: Grupo 1 (G1) com idade gestacional inferior a 34 semanas; e Grupo 2 (G2) com idade gestacional igual ou superior a 34 semanas. O desempenho da sucção nutritiva foi avaliado observando-se presença ou ausência de blocos de sucção, número e tempo das sucções nos três primeiros blocos apresentados, e presença ou ausência de coordenação entre sucção/deglutição/ respiração (S/D/R). Nenhum RNPT recebeu estimulação fonoaudiológica prévia. Resultados: O G2 apresentou, na maioria dos RNPT, presença de coordenação entre $\mathrm{S} / \mathrm{D} / \mathrm{R}$, além de melhor desempenho em relação ao número e ao tempo de sucção nos blocos de sucção quando comparado ao G1. Na correlação do número versus tempo de sucção por bloco, o G1 apresentou resultados aproximados aos do G2. Considerando-se a classificação quanto ao desenvolvimento intra-útero adequado para a idade gestacional, os RNPT do G1 apresentaram desempenho semelhante aos do G2, no primeiro e segundo blocos, observando-se queda, no G1, apenas no terceiro bloco. Conclusão: O melhor desempenho no padrão de sucção nutritiva foi apresentado pelo G2, sugerindo que a idade gestacional corrigida dos RNPT interfere diretamente nos resultados obtidos na avaliação da sucção nutritiva. Assim sendo, esta também é uma variável a ser considerada para liberação da alimentação por via oral.
\end{abstract}

Descritores: Recém-nascido; Prematuro; Idade gestacional; Alimentação; Sucção; Deglutição

\section{INTRODUÇÃO}

Os reflexos de sucção e de deglutição podem se apresentar imaturos ou até mesmo ausentes no recém-nascido pré-termo (RNPT), havendo a necessidade, nesses casos, de adequar as funções de alimentação. Na literatura, há escassez de referências que determinem o padrão de sucção do RNPT, que permitam a avaliação do que é aceitável durante o processo

Artigo extraído da monografia apresentada pela primeira autora ao Curso de Especialização em Fonoaudiologia da Universidade Federal de Santa Maria - UFSM - Santa Maria (RS), Brasil.

(1) Pós-graduanda em Distúrbios da Comunicação Humana pela Universidade Federal de Santa Maria - UFSM - Santa Maria (RS), Brasil; Professora do Curso de Fonoaudiologia da Universidade Federal de Santa Maria - UFSM - Santa Maria (RS), Brasil.

(2) Doutora; Professora dos Cursos de Graduação e Especialização em Fonoaudiologia e Mestrado em Distúrbios da Comunicação Humana da Universidade Federal de Santa Maria - UFSM - Santa Maria (RS), Brasil.

(3) Doutora; Professora do Curso de Medicina e do Mestrado em Distúrbios da Comunicação Humana da Universidade Federal de Santa Maria - UFSM - Santa Maria (RS), Brasil.

Endereço para correspondência: Raquel Coube de Carvalho Yamamoto. R. Rubem Martins Berta, 103, Bairro Camobi, Santa Maria - RS, CEP 97105-

350. E-mail: raquelcoube@yahoo.com.br

Recebido em: 16/7/2007; Aceito em: 30/8/2008 evolutivo de sucção, ou mesmo que definam as características que devem ser observadas acerca dos recém-nascidos com idade gestacional (IG) inferior a 37 semanas.

Quando apresenta uma IG ou uma idade gestacional corrigida (IGC) menor que 35 semanas, o RNPT pode apresentar imaturidade e incoordenação das funções de sucção, deglutição e respiração (S/D/R), reflexo de tosse e imaturidade gástrica ${ }^{(1)}$. Recém-nascidos com IG inferior a 34 semanas apresentam, ao nascimento, menor organização dos estados comportamentais de sono e vigília ${ }^{(2)}$. Por essas razões, faz-se necessário que os RNPTs sejam alimentados através de sonda até estarem aptos a iniciar a alimentação por via oral ${ }^{(1-2)}$.

Para que a alimentação por via oral (VO) ocorra de forma adequada, é importante que o recém-nascido tenha uma relação rítmica e coordenada entre a $\mathrm{S} / \mathrm{D} / \mathrm{R}^{(3)}$. No entanto, o RNPT com menos de 32-34 semanas de IG não apresenta sucção de forma eficiente ou, se apresenta boa sucção, ainda não é capaz de coordenar $\mathrm{S} / \mathrm{D} / \mathrm{R}$, por apresentar-se imaturo neurologicamente ${ }^{(2)}$.

Estudos mencionam parâmetros quanto à coordenação da sucção/deglutição em RNPTs e recém-nascidos a termo $(\text { RNTs })^{(4-5)}$. Em um desses estudos, encontrou-se uma taxa média de 1:1 sucção/deglutição em ambos os grupos, num mesmo período de tempo ${ }^{(4)}$. Em outra pesquisa ${ }^{(5)}$, nos RNPTs 
com IG igual ou inferior a 30 semanas, os blocos de sucção na sucção nutritiva (SN) variaram de uma a cinco sugadas; os com IG de 31 e 32 semanas variaram de seis a 15 sugadas; e os com IG maior ou igual a 33 semanas apresentaram blocos com mais de 15 sucções.

Uma pesquisa relata que a partir da $34^{\mathrm{a}}$ semana de IGC até a IGC considerada a termo, os RNPTs demonstraram diferenças significativas quanto ao número de sucções, à intensidade da pressão de sucção e à média de tempo das pausas entre os blocos $^{(6)}$.

Os RNPTs ${ }^{(3)}$ com 32 semanas de IG demonstraram incoordenação e movimento arrítmico de sucção; os com 33 semanas de IG apresentaram progressão na sucção, deflagrando aproximadamente de duas a três sucções por segundo, porém não apresentaram coordenação entre a sucção e a deglutição. Quando nascem com IG de 34 semanas, apresentam ritmo de sucção rápida e as deglutições ficam mais constantes, e a freqüência é de uma sucção por segundo.

O comportamento de alimentação em RNPT indica a duração da sucção de $0,32,0,43,0,57,0,69$ e 0,71 segundos, para as IGC de 32, 33, 3435 e 36 semanas, respectivamente ${ }^{(7)}$.

A classificação de desenvolvimento intra-útero do RN e a análise do peso de nascimento (PN) com a IG indicam se o $\mathrm{RN}$ é adequado para a IG (AIG) entre os percentis 10 e 90 da curva de referência (curvas percentuais de crescimento fetal); pequeno para a IG (PIG) abaixo do percentil 10 (abaixo de dois desvios-padrão) e RN grande para a IG (GIG) acima do percentil 90 (acima de dois desvios-padrão) ${ }^{(8)}$.

A fim de conhecer mais sobre o RNPT no momento da liberação da alimentação por VO, para uma efetiva SN, esta pesquisa analisou as características da sucção nutritiva de recém-nascidos pré-termo de diferentes idades gestacionais, após a prescrição médica para a alimentação por via oral.

\section{MÉTODOS}

\section{Aspectos Éticos}

Esta pesquisa foi realizada na Unidade de Terapia Intensiva (UTI) Neonatal de um Hospital Universitário e obteve aprovação do Comitê de Ética em Pesquisa, sob o número 108/04.

\section{Sujeitos}

A coleta dos dados ocorreu de maio a setembro do ano de 2005. Este estudo esteve composto por 79 RNPTs, os quais foram internados na UTI Neonatal de um Hospital Universitário. Destes, somente 32 RNPTs foram avaliados. Os demais não foram incluídos devido a intercorrências que impossibilitaram sua participação (conforme os critérios de exclusão), a óbito ou a recebimento da alimentação por $\mathrm{VO}$ antes da avaliação fonoaudiológica.

Os critérios de inclusão considerados foram: autorização, pelos pais e/ou responsáveis, para a participação na pesquisa, através da assinatura do Termo de Consentimento Livre e Esclarecido; idade gestacional inferior a 37 semanas; estabilidade clínica; prescrição médica para a transição da alimentação de sonda para VO.
Os critérios de exclusão considerados foram: alteração no desempenho das funções orais como malformações congênitas de cabeça e pescoço; síndromes genéticas; uso de suporte ventilatório; asfixia neonatal definida por um Apgar de $5^{\circ}$ minuto menor ou igual a 6; encefalopatia hiperbilirrubínica; e alterações no SNC diagnosticadas pela equipe médica através de exames de imagem (ultra-sonografia e tomografia computadorizada de crânio).

A amostra foi dividida conforme a IGC dos RNPTs no momento da avaliação fonoaudiológica, sendo constituídos dois grupos: Grupo 1 (G1), com seis RNPTs de IGC inferior a 34 semanas; e Grupo 2 (G2) com 26 RNPTs de IGC igual ou superior a 34 semanas. No segundo momento da pesquisa, foram separados da amostra apenas os RNPTs classificados como AIG, excluindo-se os RNs classificados como PIG e GIG. Todos os RNPTs que fizeram parte desta pesquisa não receberam qualquer estimulação fonoaudiológica prévia.

\section{Material}

Inicialmente, foram coletados os dados dos prontuários a fim de obter informações sobre as características dos recémnascidos (peso ao nascimento e no momento da avaliação, IG, IGC e classificação referente ao desenvolvimento intra-útero - PIG, AIG e GIG). Na avaliação fonoaudiológica, utilizou-se protocolo $^{(9-11)}$ que constou de análise específica dos reflexos orais (busca, sucção, deglutição), sistema sensório-motororal (lábios, língua, bochechas, mandíbula, palato duro e palato mole), morfologia de faces e assimetria. Os RNPTs que apresentaram qualquer alteração morfológica não foram incluídos na pesquisa.

\section{Procedimentos}

A avaliação fonoaudiológica ocorria após a liberação da VO pela equipe médica responsável pelo RNPT, e sempre dez minutos antes do horário estimado para a alimentação. $\mathrm{O}$ RNPT era posicionado em decúbito dorsal elevado, aproximadamente num ângulo de $45^{\circ}$, com uma postura organizada.

A avaliação da SN era realizada com a introdução da mamadeira na cavidade oral do RNPT. A partir daí, analisavam-se detalhadamente os primeiros três blocos da SN no RNPT, observando-se presença ou ausência de blocos de sucção, número e tempo das sucções nos três primeiros blocos apresentados pelo RNPT avaliado e presença ou ausência de coordenação entre S/D/R. A avaliação foi filmada, e os dados foram analisados posteriormente, mediante a observação da imagem de fita VHS por três fonoaudiólogas com experiência nesta área. Cada profissional analisou as filmagens individualmente e, em seguida, os dados foram tabulados.

\section{Análise dos dados}

Os dados foram analisados, tomando-se como critério os resultados comparativos obtidos em relação ao peso na ocasião do nascimento e no momento da avaliação fonoaudiológica, à idade gestacional de nascimento e à IGC. A SN foi comparada quanto à coordenação da $S / D / R$ e às características apresenta- 
das nos três primeiros blocos de sucção, referentes ao número e ao tempo de sucção em cada bloco. Posteriormente, foi feita a correlação desses valores.

$\mathrm{Na}$ correlação realizada entre o número de sucções por blocos de sucção nutritiva e o seu respectivo tempo, foram excluídos os RNPTs que não apresentaram blocos de sucção. Assim, no G1, apenas um RNPT foi excluído para os três blocos analisados e, no G2, foram excluídos dois RNPTs no primeiro e segundo blocos de sucção, chegando ao total de quatro RNPTs no terceiro bloco, por não apresentarem sucção ou por terem apresentado tempo de sucção inferior a um segundo.

Os resultados foram analisados e tabulados utilizando-se o software estatístico STATA, versão 5.0 (1998), sendo usado o teste paramétrico de variância ANOVA, na análise das variáveis expressas em médias e desvio padrão, e o Qui-quadrado, na análise das variáveis expressas em percentuais. Utilizou-se, também, o coeficiente de correlação de Spearman, para o cálculo das correlações entre as variáveis estudadas.

\section{RESULTADOS}

A Tabela 1 apresenta a caracterização dos G1 e G2 referente a: peso ao nascimento; peso na data da avaliação; idade gestacional; e idade gestacional corrigida.

A Tabela 2 apresenta características quanto à $\mathrm{SN}$ dos G1 e G2 nas seguintes variáveis: presença de coordenação entre S/D/R; número e tempo de sucção nos três primeiros blocos.

Durante a avaliação da SN, alguns dos RNPT da amostra estudada não apresentaram sucção. Estes foram excluídos para a contagem do número e do tempo de sucção por bloco, ficando, no G1, um total de cinco RNPT na amostragem dos três primeiros blocos de sucção; 24 RNPT no G2, para o primeiro e segundo blocos de sucção; e 22 RNPTs no G2 para o terceiro bloco.

A Figura 1 apresenta a correlação nos três primeiros blocos de sucção no G1 entre o número de sucções por blocos e o tempo dos blocos na SN.

Na Figura 1 estão dispostos os resultados da correlação do número de sucções (B1, B2, B3) por blocos da sucção nutritiva e o tempo $(\mathrm{T} 1, \mathrm{~T} 2, \mathrm{~T} 3)$ utilizado para a realização dessas sucções.

A Figura 2 apresenta a correlação, no G2, entre o número de sucções por blocos da $\mathrm{SN}$ e o seu tempo nos três blocos avaliados.

Na Tabela 3 observa-se a divisão da amostra quanto ao desenvolvimento intra-útero na classificação de AIG. Todos os RNPTs representados no G1 $(n=6)$ estavam classificados como AIG e, na amostra de 26 RNPTs do G2, apenas 14 foram classificados como AIG. Foram analisados os dados sobre o peso, ao nascimento e na avaliação fonoaudiológica, assim como os dados obtidos nos três primeiros blocos de sucção, na avaliação da sucção nutritiva dos RNPTs que apresentaram tais blocos.

Nos dados referentes ao bloco de sucção, na Tabela 3, foram excluídos os RNPTs que não apresentaram bloco de sucção no momento da avaliação fonoaudiológica da $\mathrm{SN}$, permanecendo, assim, um total de cinco RNPTs no G1 e de 12 RNPTs no G2.

Quanto aos resultados encontrados no G1, após a divisão quanto ao desenvolvimento intra-útero, nota-se que os dados são iguais aos já obtidos para o G1. Isso se deve ao fato de todos

Tabela 1. Caracterização dos grupos quanto ao peso de nascimento, peso no dia da avaliação, idade gestacional, e idade gestacional corrigida

\begin{tabular}{lccc}
\hline Variáveis & G1 & G2 & Valor de $p$ \\
\hline Peso de nascimento $(\mathrm{g})^{*}$ & $1.527( \pm 185)$ & $1.665( \pm 576)$ & 0,5734 \\
\hline Peso na avaliação $(\mathrm{g})^{*}$ & $1.788( \pm 79)$ & $1.881( \pm 461)$ & 0,6294 \\
\hline Idade gestacional (semanas) $^{*}$ & $31( \pm 1,2)$ & $33,2( \pm 2,0)$ & $0,0070^{\#}$ \\
\hline Idade gestacional corrigida (semanas) $^{*}$ & $33( \pm 0)$ & $35,3( \pm 1,3)$ & $0,0002^{\#}$ \\
\hline
\end{tabular}

Legenda: *Valores expressos em média e desvio padrão; "Valores estatisticamente significantes

Tabela 2. Características dos grupos estudados quanto a sucção nutritiva nas variáveis e presença de coordenação entre sucção/deglutição/ respiração $(\mathrm{S} / \mathrm{D} / \mathrm{R})$; número e tempo de sucção

\begin{tabular}{|c|c|c|c|}
\hline Variáveis & G1 & G2 & Valor de $p$ \\
\hline Presença de coordenação S/D/R (\%) & $67 \%$ & $85 \%$ & 0,310 \\
\hline \multicolumn{4}{|l|}{ Número de sucções (sucção)* } \\
\hline $1^{\circ}$ bloco & $9,8( \pm 10,0)$ & $12,2( \pm 14,4)$ & 0,6871 \\
\hline $2^{\circ}$ bloco & $3,4( \pm 2,9)$ & $6,4( \pm 7,3)$ & 0,3605 \\
\hline $3^{\circ}$ bloco & $1,6( \pm 1,3)$ & $7,7( \pm 10,1)$ & 0,1862 \\
\hline \multicolumn{4}{|l|}{ Tempo das sucções (segundos)* } \\
\hline $1^{\circ}$ bloco & $10,4( \pm 9,8)$ & $12,6( \pm 14,9)$ & 0,7184 \\
\hline $2^{\circ}$ bloco & $5,4( \pm 4,8)$ & $7,3( \pm 8,1)$ & 0,5939 \\
\hline $3^{\circ}$ bloco & $2( \pm 1,7)$ & $8,5( \pm 9,6)$ & 0,1751 \\
\hline
\end{tabular}

Legenda: *Valores expressos em média e desvio padrão; \# Valores estatisticamente significantes; S/D/R = sucção/deglutição/respiração 


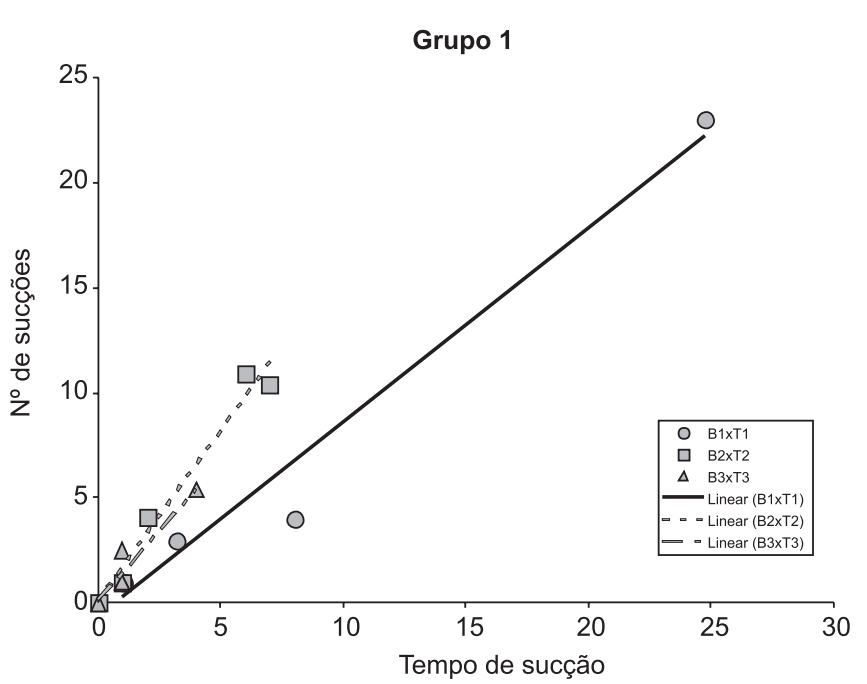

Figura 1. Correlação entre os três blocos de sucção quanto ao número e tempo das sucções do Grupo 1

Legenda: $\mathrm{B} 1=$ número de sucções no primeiro bloco; $\mathrm{B} 2$ = número de sucções no segundo bloco; B3 = número de sucções no terceiro bloco; T1 = tempo de sucção no primeiro bloco; T2 = tempo de sucção no segundo bloco; T3 = tempo de sucção no terceiro bloco.

Correlação: $\mathrm{B} 1 x \mathrm{~T} 1$ : $\mathrm{R}=0,9654$; $\mathrm{B} 2 \times \mathrm{T} 2$ : $\mathrm{R}=0,9744$; $\mathrm{B} 3 \times \mathrm{T} 3$ : $\mathrm{R}=0,9682$

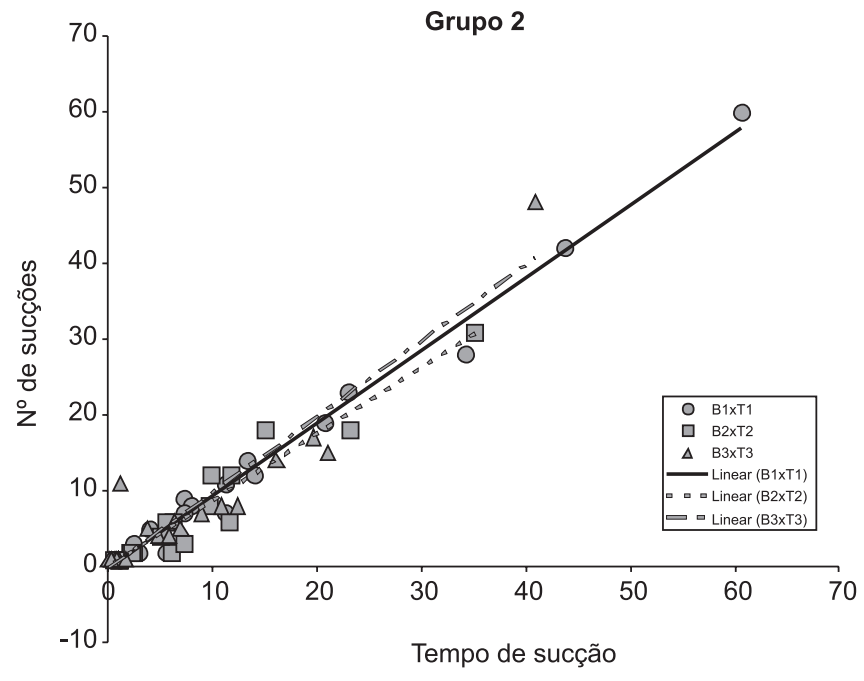

Figura 2. Correlação entre os três blocos de sucção quanto ao número e tempo das sucções do Grupo 2

Legenda: $\mathrm{B} 1$ = número de sucções no primeiro bloco; $\mathrm{B} 2$ = número de sucções no segundo bloco; B3 = número de sucções no terceiro bloco; T1 = tempo de sucção no primeiro bloco; T2 = tempo de sucção no segundo bloco; T3 = tempo de sucção no terceiro bloco.

Correlação: $B 1 x T 1$ : $R=0,9887$; $B 2 x T 2: R=0,9639$; $B 3 x T 3$ : $R=0,9461$

Tabela 3. Características dos recém-nascidos pré-termos classificados como adequados a idade gestacional ao nascimento quanto suas características gerais e característica apresentada nos blocos de sucção avaliados

\begin{tabular}{|c|c|c|c|}
\hline Variáveis & G1-AIG & G2-AIG & Valor de $p$ \\
\hline Peso de nascimento $(\mathrm{g})^{*}$ & $1.527( \pm 185)$ & $1.653( \pm 495)$ & 0,5596 \\
\hline Peso na avaliação $(\mathrm{g})^{*}$ & $1.788( \pm 79)$ & $1.886( \pm 436)$ & 0,5994 \\
\hline Idade gestacional (semanas) ${ }^{*}$ & $31( \pm 1,2)$ & $32,6( \pm 2,1)$ & $0,0265^{\#}$ \\
\hline Idade gestacional corrigida (semanas)* & $33( \pm 0)$ & $35,2( \pm 1,3)$ & $0,0025^{\#}$ \\
\hline \multicolumn{4}{|l|}{ Número de sucções (sucção)* } \\
\hline $1^{\circ}$ bloco & $9,8( \pm 10,0)$ & $10,3( \pm 11,7)$ & 0,9302 \\
\hline $2^{\circ}$ bloco & $3,4( \pm 2,9)$ & $4,7( \pm 4,9)$ & 0,5999 \\
\hline $3^{\circ}$ bloco & $1,6( \pm 1,3)$ & $4,6( \pm 4,6)$ & 0,1850 \\
\hline \multicolumn{4}{|l|}{ Tempo das sucções (segundos) ${ }^{*}$} \\
\hline $1^{\circ}$ bloco & $10,4( \pm 9,8)$ & $11,1( \pm 12,0)$ & 0,9124 \\
\hline $2^{\circ}$ bloco & $5,4( \pm 4,8)$ & $5,8( \pm 6,6)$ & 0,8964 \\
\hline $3^{\circ}$ bloco & $2( \pm 1,7)$ & $5,6( \pm 5,7)$ & 0,1970 \\
\hline
\end{tabular}

Legenda: *Valores expressos em média e desvio padrão; " Valores estatisticamente significantes; AIG = adequado para a idade gestacional

os RNPTs deste grupo terem nascido com um desenvolvimento intra-útero classificado como AIG. Por essa razão, o seu gráfico de correlação é o mesmo representado pela Figura 1.

A Figura 3 representa a correlação do grupo de maior IGC que, no dia da avaliação, apresentava seu desenvolvimento intra-útero adequado para sua idade gestacional. Estão dispostos dados dos 12 RNPTs AIG, quanto ao número e tempo de sucção, nos três primeiros blocos de sucção apresentados por esses RNPTs.

\section{DISCUSSÃO}

Os grupos foram caracterizados e comparados quanto a peso, idade gestacional, idade gestacional corrigida e, na SN, quanto ao número e tempo das sucções nos três primeiros blo- cos de sucção, coordenação da S/D/R na primeira alimentação por $\mathrm{VO}$, assim como quanto às características do desenvolvimento intra-útero apresentadas pelo RNPT ao nascimento.

Nas características gerais dos dois grupos de RNPT (Tabela 1), pode-se constatar que os resultados apresentaram diferença estatisticamente significativa quanto à IG $(\mathrm{p}=0,0070)$ e à IGC $(\mathrm{p}=0,0002)$. Porém, nas variáveis peso ao nascimento e peso na primeira avaliação, não foi constatada diferença estatisticamente significativa entre os grupos, ou seja, apresentaram médias próximas na variável peso ao nascimento, com $1527 \mathrm{~g}$ $( \pm 185)$ no G1 e $1665 \mathrm{~g}( \pm 576)$ no G2, e média de peso no dia da primeira avaliação de $1788 \mathrm{~g}( \pm 79)$ no G1 e $1881 \mathrm{~g}( \pm 461)$ no G2, mesmo este sendo de diferente IG.

Um estudo ${ }^{(12)}$ que teve como propósito examinar o efeito da sucção não-nutritiva ( $\mathrm{SNN}$ ) sobre a respiração, da SN e das 


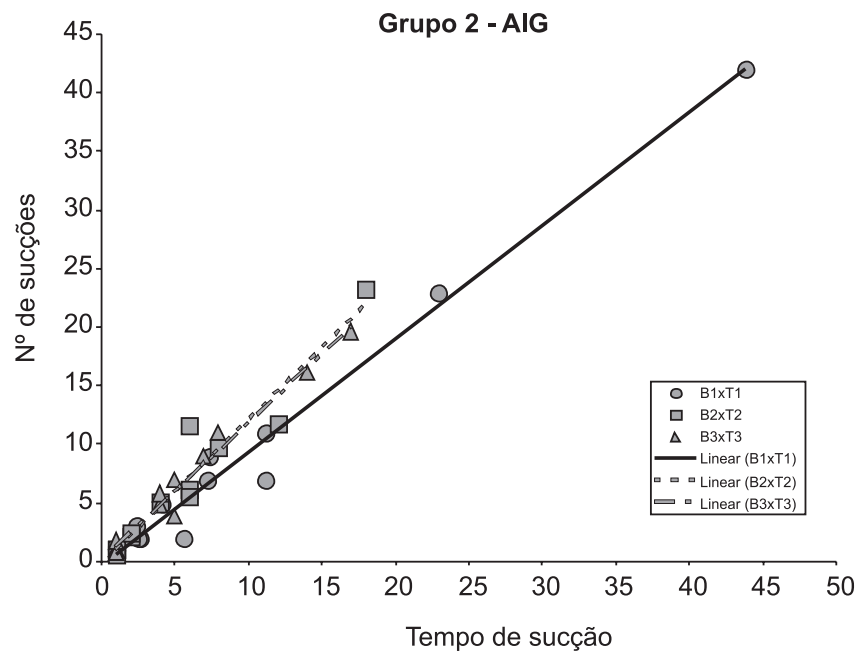

Figura 3. Correlação entre os blocos de sucção dos recém-nascidos pré-termos classificados como adequados a idade gestacional ao nascimento do Grupo 2

Legenda: $\mathrm{B} 1$ = número de sucções no primeiro bloco; $\mathrm{B} 2$ = número de sucções no segundo bloco; B3 = número de sucções no terceiro bloco; T1 = tempo de sucção no primeiro bloco; T2 = tempo de sucção no segundo bloco; T3 = tempo de sucção no terceiro bloco; $\mathrm{AIG}=$ adequado para a idade gestacional

Correlação: B1xT1: R=0,9874; B2xT2: $R=0,9734$; B3xT3: $R=0,9846$.

características comportamentais da alimentação por via oral, verificou que, na primeira alimentação por via oral, a liberação ocorria em média com 33,5 semanas de IG em RNPT com peso médio de $1594 \mathrm{~g}$, no dia da avaliação. A IG não deve ser considerada isoladamente como critério de prontidão para a sucção, mas a maioria dos RNPT recebeu liberação para VO em aproximadamente 34 semanas de IGC, apresentando, neste momento, condições clínicas necessárias para liberação ${ }^{(13)}$. Sendo assim, em geral, os requisitos básicos para a liberação da alimentação por via oral dos RNPTs seriam estabilidade clínica, peso e idade gestacional adequados ${ }^{(12-14)}$.

As variáveis referentes a peso ao nascimento e peso na primeira avaliação apresentaram resultados aproximados. Isto provavelmente ocorreu pela diferença encontrada quanto à classificação usada para caracterizar o recém-nascido no que se refere ao desenvolvimento intra-útero. No G2, dez RNPTs apresentavam-se pequenos para a IG (PIG), dois grandes (GIG) e 14 adequados (AIG).

No entanto, pode-se perceber que os RNPTs, tanto do G1 quanto do G2, não apresentaram grande diferença em relação ao peso no momento da avaliação fonoaudiológica. Isso sugere que a IG seria um dado importante a ser observado na liberação da alimentação por VO, uma vez que a IGC seria fator para justificar a maturidade para a SN, ao invés do peso do RNPT.

Em relação à coordenação S/D/R (Tabela 2), pôde-se observar que $67 \%$ dos RNPTs apresentaram coordenação S/D/R no G1, enquanto que no G2 a maioria (85\%) apresentou coordenação $\mathrm{S} / \mathrm{D} / \mathrm{R}$. Estes dados não demonstraram diferença estatisticamente significante $(\mathrm{p}=0,310)$, sendo que o grupo de maior IG, no momento da avaliação, apresentou melhor desempenho relacionado à coordenação $\mathrm{S} / \mathrm{D} / \mathrm{R}$. Provavelmente, a diferença estatisticamente significativa não ocorreu devido ao tamanho da amostra do G1 (composta por apenas seis RNPTs), caracterizando um número muito pequeno de sujeitos para se fazer comparação com o G2. Porém, nota-se que o grupo de maior IGC na avaliação apresenta-se melhor organizado quanto aos aspectos de coordenação S/D/R, demonstrando que o G2 está mais apto que o G1 para iniciar a alimentação por VO.

Os resultados obtidos quanto à coordenação $\mathrm{S} / \mathrm{D} / \mathrm{R}$ na $\mathrm{SN}$ denotam que nessa variável os sujeitos de maior IG têm desempenho satisfatório. Infere-se, a partir disso, que a maturação neuromuscular apresentada pelos sujeitos com maior IGC pode ser fator determinante para que os RNPTs alcancem melhor desempenho no momento da liberação da SN. O crescente equilíbrio entre o ritmo de sucção e a relação entre sucção/deglutição pode ser descrito como sendo um sinal de maturidade do RN no decorrer do processo de alimentação por $\mathrm{VO}^{(3)}$.

Outra pesquisa ${ }^{(15)}$ realizada com a mesma amostra do presente estudo, dividiu tal amostra em dois grupos: quanto à presença ou à ausência dos reflexos de busca, sucção e deglutição. Os resultados do desempenho dos RNPTs na avaliação da $\mathrm{SN}$, quanto ao ritmo de sucção, indicaram que, no grupo com reflexos presentes, $75 \%$ dos RNPTs tiveram ritmo de sucção rápido, enquanto que, no grupo com reflexos ausentes, apenas 41,7\% apresentaram ritmo rápido. Estes resultados não demonstraram diferença estatisticamente significativa e diferem dos resultados encontrados na SNN, uma vez que o ritmo de sucção rápido caracterizou $90 \%$ dos RNPTs pertencentes ao grupo com reflexos presentes e $33,3 \%$ dos integrantes do grupo com reflexos ausentes, havendo diferença estatisticamente significativa em relação ao ritmo $(\mathrm{p}<0,001)$.

Concluindo, a presença dos três reflexos orais adaptativos na SNN possibilita manter um padrão rápido de sucção. Porém, na SN, o RNPT não consegue manter um padrão rápido de sucção como o apresentado na SNN, em função da presença do leite, realizando o movimento de sucção de forma mais lenta, uma vez que ele precisa coordenar a S/D/R.

Os resultados encontrados quanto à coordenação da $S / D / R$ (Tabela 2) podem se referir a uma imaturidade neurológica apresentada pelo RN de menor IG. Outra pesquisa ${ }^{(2)}$ corrobora as descobertas do presente estudo, uma vez que os RNPTs do G1, com IGC inferior a 34 semanas, foram os que apresentaram menor porcentagem quanto à coordenação $S / D / R$ no momento da SN.

Em pesquisa realizada com 25 RNPTs e 25 RNTs avaliados entre o $3^{\circ}$ e o $5^{\circ}$ dia após liberação para via oral no seio materno, copo ou mamadeira, os autores verificaram que $84 \%$ dos RNTs apresentaram coordenação S/D/R, ou seja, demonstraram modo sincronizado de respirar, fazendo pausas espontâneas para deglutir, não soltando a mama ou o bico da mamadeira nas pausas e retomando, espontaneamente, a sucção após a pausa. Desempenho inferior foi obtido nos RNPTs avaliados ${ }^{(16)}$.

Outras pesquisas realizadas concluíram que o fato de $16 \%$ dos RNTs pesquisados não terem apresentado coordenação da S/D/R presente confirma o achado de não ser suficiente nascer a termo para coordenar tais funções ${ }^{(17-18)}$.

Em uma pesquisa baseada no estudo ultra-sonográfico da sucção e deglutição de RNTs, foram observados os movimen- 
tos de sucção, deglutição e respiração, durante a alimentação no peito e na mamadeira, e verificado que a sucção pode ocorrer isoladamente ou em combinação com a deglutição. Já a deglutição não ocorre isoladamente, pois em crianças menores (menores que a termo) associou-se, invariavelmente, a pausa na respiração ${ }^{(19)}$.

Um estudo realizado $^{(20)}$ com $13 \mathrm{RNs}$, entre 14 e 49 dias de idade pós-natal, com o objetivo de verificar o padrão da $\mathrm{SN}$ e SNN, e de analisar a influência da sucção no processo da alimentação, constatou que 53,8\% dos RNs apresentaram sucção normal, $30,8 \%$, sucção alterada e, em 15,4\%, não foi possível concluir a triagem, pois o RN apresentou sinais de estresse. Este estudo concluiu que o número de RNs com sucção alterada foi significativo, sugerindo que o procedimento de triagem fonoaudiológica deve ser implantado como ação complementar, junto aos programas de incentivo ao aleitamento materno, a fim de prevenir alterações fonoaudiológicas ${ }^{(20)}$. Se não identificadas e não sanadas estas alterações, como, por exemplo, padrão inadequado de respiração e postura incorreta da língua, o RN pode ser levado ao desmame precoce, o que pode acarretar a ruptura do desenvolvimento motor-oral adequado, alterando a postura e a força dos órgãos fonoarticulatórios e prejudicando as funções de mastigação, deglutição, respiração e articulação dos sons da fala ${ }^{(21)}$.

Ao analisar o número de sucção ocorrido nos três primeiros blocos realizados pelo RNPT (Tabela 2), pode-se observar que o G1 apresentou os três primeiros blocos de sucção de maneira desorganizada, com uma média de 9,8 sucções $( \pm 10,0)$ no primeiro bloco, 3,4 sucções $( \pm 2,9)$ no segundo, e 1,6 $( \pm 1,3)$ no terceiro. Os RNPTs do G2 mostraram-se melhor organizados quanto aos números de sucção de um bloco para o outro, apresentando uma média de $12,6( \pm 14,6)$ no primeiro bloco, 6,6 $( \pm 7,5)$ no segundo, e 7,9 $( \pm 10,2)$ no terceiro bloco. O estudo comparativo por blocos de sucção não demonstrou diferença estatisticamente significante entre os grupos estudados (Bloco 1: $\mathrm{p}=0,6871$; Bloco 2: $\mathrm{p}=0,3605$; e Bloco 3: $\mathrm{p}=0,1862$ ), provavelmente devido ao número de sujeitos da amostra do G1. Contudo, as médias do número de sucção denotam que o grupo de menor IG fadigou-se no decorrer dos três primeiros blocos, como se pode observar através da queda brusca do número de sucções por bloco apresentado.

O tempo de sucção também foi observado nos três blocos de sucção avaliados. No primeiro bloco de sucção do G1, o tempo de sucção teve uma média de 10,4 segundos $( \pm 9,8)$; no segundo bloco de 5,4 segundos $( \pm 4,8)$; e no terceiro de 2,0 segundos $( \pm 1,7)$. No G2, constatou-se uma média de tempo de sucção por bloco de 13,0 segundos $( \pm 15,1), 7,5$ segundos $( \pm 8,2)$ e 8,1 segundos $( \pm 9,6)$ para o primeiro, o segundo e o terceiro blocos de sucção, respectivamente.

Quanto ao bloco de sucção, no que se refere ao número e ao tempo de sucção, um estudo ${ }^{(5)}$ constatou que a SN das crianças nascidas com IG igual ou inferior a 30 semanas variou de uma a cinco sugadas; em RNs nascidos com IG de 31 e 32 semanas, variou de seis a 15; e em três crianças com IG maior ou igual a 33 semanas, verificaram-se mais de 15 sugadas por blocos.

Nos resultados desta pesquisa (Tabela 2), pode-se observar que, no primeiro bloco de sucção, o G2 apresentou número elevado de sucções sem apresentar alterações, como hipóxia. Isso contraria a descrição que indica que, quando o RN apresenta blocos de sucções com mais de oito sucções por bloco, pode ocorrer apnéia, provocando hipóxia ${ }^{(22)}$.

Os resultados referentes à média do número de sucção por bloco e seu respectivo tempo indicam que, no G1, os RNPTs apresentaram, no primeiro, segundo e terceiro blocos, 0,94, 0,62 e 0,80 sucções/segundo, respectivamente. Já o G2 apresentou, no primeiro, segundo e terceiro blocos, os valores respectivos de 0,97, 0,88 e 0,97 sucções/segundo, aproximando-se dos resultados encontrados em outra pesquisa ${ }^{(3)}$.

Em um outro estudo ${ }^{(6)}$ foi constatado que RNPTs, desde a $34^{\mathrm{a}}$ semana de IGC até serem considerados a termo, apresentaram diferenças significativas quanto ao número de sucções, à intensidade da pressão de sucção e à média de tempo das pausas entre os blocos.

Outra pesquisa ${ }^{(23)}$ que analisou o padrão de sucção nutritiva em RNPTs de muito baixo peso, com média de IG de 29,5 semanas e de peso de $1466 \mathrm{~g}$, verificou que o número total de sucções durante a alimentação foi alterado com o aumento da IG, apresentando, assim, um número de 118,28 sucções $( \pm 62,5)$, na $32^{\mathrm{a}}$ semana, e de 182,75 sucções $( \pm 104,6)$, na $36^{\mathrm{a}}$ semana. O tempo entre os blocos diminuiu de 13,95 segundos, na $32^{\mathrm{a}}$ semana, para 6,95 , na $36^{\mathrm{a}}$ semana.

O recém-nascido apresenta uma melhora gradativa da retirada do leite da mamadeira com o aumento da IG, chegando ao seu melhor desempenho quando alcança a $40^{\mathrm{a}}$ semana $^{(24)}$. Um padrão de sucção é considerado eficiente quando consegue ingerir todo o volume prescrito em menos de $3 \mathrm{ml} / \mathrm{min}^{(9,11)}$. Tal resultado pode estar implicitamente ligado à coordenação S/D/R, apresentada pelo RNPT, e pela maturidade na capacidade da sucção nutritiva, esta adquirida com uma IGC de 34 semanas.

Ao analisar a Figura 1, pode-se observar que os RNPTs do G1 mantiveram uma forte correlação entre o número de sucções por blocos de sucção e o seu respectivo tempo (o coeficiente de correlação para o primeiro, segundo e terceiro blocos de sucção foi de $0,9654,0,9744,0,9682$, respectivamente). Isso demonstra que, mesmo nunca tendo recebido alimentação por VO, os RNPTs de menor IG apresentaram, no primeiro bloco de sucção, uma boa correlação com o tempo de sucção.

Porém, ao analisar os dados de maneira isolada, observase que todos os RNPTs tiveram um decréscimo semelhante do primeiro para o segundo e terceiro blocos de sucção, permanecendo, assim, uma forte correlação entre o número de sucção por bloco e o tempo de sucção. Constatou-se que, mesmo apresentando queda significativa dos dados relativos ao bloco de sucção, todos os RNPTs deste grupo decresceram seu número e tempo de sucção, no decorrer dos três blocos estudados.

Na Figura 2 estão representadas as correlações entre os blocos de sucção, tempo e número de sucções apresentadas pelo G2 nos três primeiros blocos estudados na avaliação da SN. Mesmo que este grupo tenha apresentado uma discreta diminuição da correlação no segundo bloco de sucção, a correlação mostrou-se positiva. $O$ valor do coeficiente de correlação encontrado no primeiro, segundo e terceiro blocos de sucção foi de $0,9887,0,9639$ e 0,9461 , respectivamente. 
Observando-se as Figuras 1 e 2 verifica-se que o desempenho entre ambos os grupos foi semelhante. Ao analisar os dados da variável 'bloco de sucção' (Tabela 2), nota-se que o G2 apresentou melhor organização quanto ao número e tempo de sucções por bloco, indicando, assim, que o grupo de maior idade gestacional encontra-se em melhores condições neurofisiológicas para receber a alimentação por VO.

Com isso, pode-se supor que a sensibilidade intra-oral e a integridade dos reflexos apresentaram-se defasados no grupo de menor IG (G1), talvez devido ao grande período de tempo sem estímulo de sucção ou devido ao contato constante da sonda orogástrica com a cavidade oral, sendo o grupo de menor IG, provavelmente, o que permaneceu mais tempo internado sem estímulo intra-oral, em função da prematuridade. Neste caso, a IGC dos RNPTs avaliados mostrou que os de menor IG apresentam desempenho inferior na correlação entre o número e o tempo de sucção.

Essa discussão concorda com outros autores ${ }^{(21,25-28)}$, que descrevem o RNPT como apresentando imaturidade global, a qual atinge também o sistema estomatognático do recém-nascido; e, com isso, a função de sucção e a alimentação por VO ocorrem de maneira ineficiente. Outros estudos ${ }^{(4,28-30)}$ também compartilham destas conclusões, ressaltando que os RNPTs com IGC inferior a 34 semanas podem apresentar uma desorganização da SN devido à falta de estímulos sensoriais durante a alimentação, pois esta se realiza através da sonda orogástrica e, assim, afeta o desenvolvimento motor-oral do bebê.

Na Tabela 3 estão divididos os grupos considerando a classificação do desenvolvimento intra-útero que os RNPTs apresentavam ao nascimento. O G1-AIG permaneceu com a mesma amostra do G1, pois todos os RNPTs com IGC inferior a 34 semanas, no momento da avaliação fonoaudiológica, foram classificados como AIG. No grupo de maior IG (G2-AIG), observa-se que o número de sujeitos da amostra diminuiu para 14 RNPTs classificados como AIG ao nascimento. Suas características, quanto a peso de nascimento, peso no dia da avaliação, IG e IGC, apresentaram dados aproximados ao da amostra geral.
Comparando os dados referentes ao bloco de sucção (Tabela 3), observou-se que os RNPTs de ambos os grupos apresentaram resultados mais aproximados para o número e o tempo de sucção, no primeiro e segundo blocos de sucção, destoando os resultados quanto ao número e tempo de sucção no terceiro bloco. Percebe-se que o G1-AIG só apresentou discrepância nas variáveis número e tempo de sucção no terceiro bloco de sucção. Os dados, ao serem analisados, não apresentaram diferença estatisticamente significativa, provavelmente pela diferença no número de sujeitos entre o G1 e o G2. Como o grupo de menor IG (G1-AIG) permaneceu o mesmo do grupo anterior (G1), manteve-se o gráfico de dispersão e suas correlações para representar também este grupo.

Ao dividir a amostra quanto à classificação de desenvolvimento intra-útero, observou-se que, tanto o G1-AIG quanto o G2-AIG apresentam correlações positivas entre o número e o tempo de sucção por bloco realizado. Na análise das médias realizadas em cada bloco, observou-se que estas eram semelhantes no primeiro e segundo blocos de sucção.

\section{CONCLUSÃO}

Pode-se concluir que a idade gestacional dos RNPTs interfere diretamente nos resultados obtidos na avaliação da SN, demonstrando que o grupo de maior IGC foi o que apresentou coordenação S/D/R na maioria dos RNPTs, além de melhor desempenho em relação ao número de sucções e maior tempo de sucção na maioria dos blocos de sucção. Na correlação entre o número de sucção por bloco e tempo de sucção, o grupo dos RNPTs de maior IGC apresentou resultados muito próximos aos resultados apresentados pelo G1, porém com sutil melhora no desempenho.

$\mathrm{Na}$ amostra classificada como adequada para a idade gestacional, os RNPTs de ambos os grupos obtiveram desempenho semelhante nos dois primeiros blocos de sucção, sendo que o G1-AIG, na avaliação do terceiro bloco, provavelmente apresentou fadiga e, conseqüentemente, queda em seu desempenho durante a SN.

\begin{abstract}
Purpose: To analyze the characteristics of nutritive sucking in preterm infants of different gestational ages after the medical prescription for oral feeding. Methods: The sample comprised 32 preterm infants who were evaluated at the time they were released for oral feeding. The subjects were divided into two groups: Group 1 (G1), with gestational ages below 34 weeks; and Group 2 (G2), with gestational ages equal or above 34 weeks. Performance during nutritive sucking was evaluated by observing presence or absence of suction blocks, number and duration of suctions in the first three blocks, and presence or absence of suction/swallowing/breathing (S/S/B) coordination. None of the preterm infants in this sample had previously received oral motor sensory stimulation. Results: Group 2 showed, in most preterm infants, the presence of S/S/B coordination, besides a better performance regarding number and duration of sucking blocks, when compared to Group 1. Regarding correlation between number and duration of sucking by block, G1 and G2 had similar results. Considering the intrauterine development of the subjects suitable to the gestational age, G1 and G2 presented similar performances both in the first and second sucking blocks. A decrease was observed in G1 in the third block. Conclusion: Group 2 showed better development of the nutritive sucking pattern, suggesting that the corrected gestational age of preterm infants interfered in the results obtained at the nutritive sucking evaluation. Therefore, this is also a relevant variable to be considered for oral feeding release.
\end{abstract}

Keywords: Infant, newborn; Infant, premature; Gestational age; Feeding; Suction; Deglutition 


\section{REFERÊNCIAS}

1. Levy DS. Atuação fonoaudiológica com recém-nascidos de alto risco. In: Ribas LP. Anuário de fonoaudiologia. Novo Hamburgo: Feevale; 2003. p.115-29.

2. McCain GC, Gartside PS, Greenberg JM, Lott JW. A feeding protocol for healthy preterm infants that shortens time to oral feeding. J Pediatr. 2001;139(3):374-9. Comment in: Evid Based Nurs. 2002;5(3):74.

3. Gewolb IH, Vice FL, Schwietzer-Kenney EL, Taciak VL, Bosma JF. Developmental patterns of rhythmic suck and swallow in preterm infants. Dev Med Child Neurol. 2001;43(1):22-7.

4. Lau C, Smith EO, Schanler RJ. Coordination of suck-swallow and swallow respiration in preterm infants. Acta Pediatr. 2003;92(6):721-7.

5. Nyqvist KH, Sjödén P, Ewald U. The development of preterm infants' breastfeeding behavior. Early Hum Dev. 1999;55(3):247-64.

6. Medoff-Cooper B, McGrath JM, Bilker W. Nutritive sucking and neurobehavioral development in preterm infants from 34 weeks PCA to term. MCN Am J Matern Child Nurs. 2000;25(2):64-70.

7. Mizuno K, Ueda A. The maturation and coordination of sucking, swallowing, and respiration in preterm infants. J Pediatr. 2003;142(1):3640. Comment on: J Pediatr. 1997;130(4):561-9.

8. Falcão MC. Terapia nutricional do recém-nascido. In: Hernandez AM, organizadora. Conhecimentos essenciais para atender bem o neonato. São José dos Campos: Pulso Editorial; c2003. p.35-46.

9. Xavier C. Assistência à alimentação de bebês hospitalizados. In: Basseto MCA, Brock R, Wajnsztejn R. Neonatologia: um convite à atuação fonoaudiológica. São Paulo: Lovise; 1998. p.255-75.

10. Neiva FCB. Proposta de um formulário de avaliação de sucção de recémnascidos. Pró-Fono. 2000;12(2):113-9.

11. Medeiros AMC, Oliveira KRF, Bittencourt AB, Alves PCL, Araripe ABS, Cardoso MFR, Hashimoto OS. Caracterização da atuação em berçário neonatal: uma visão fonoaudiológica. In: Marchesan IQ, Zorzi J, organizadores. Tópicos em fonoaudiologia 2002/2003. Rio de Janeiro: Revinter; 2003. p.293-308.

12. Pickler RH, Reyna BA. A descriptive study of bottle-feeding opportunities in preterm infants. Adv Neonatal Care. 2003;3(3):13946

13. Caetano LC, Fujinaga CI, Scochi CGS. Sucção não nutritiva em bebês prematuros: estudo bibliográfico. Rev Latinoam Enferm. 2003;11(2):232-6.

14. Almeida ST, Delgado SE, Késke MC. A eficiência da intervenção fonoaudiológica em UTI neonatal na assistência à alimentação. PróFono. 1998;10(1):34-9.

15. Prade LS. Recém-nascido pré-termo: critérios para introdução da alimentação por via oral [dissertação] Santa Maria: Universidade Federal de Santa Maria; 2006.
16. Oliveira TA, Delgado SE. Técnicas de alimentação e suas repercussões oromotoras nos bebês de risco. Rev Soc Bras Fonoaudiol. 2005;10(4):218-25.

17. Araujo KCS, Poyart MCMS, Barros MRM, Lopes JMA, Chiappetta ALML. Os efeitos do controle motor oral na alimentação de prematuros em unidades de terapia intensiva neonatal. Rev CEFAC. 2004;6(4):1827.

18. Sanches MTC. Manejo clínico das disfunções orais na amamentação. J Pediatr (Rio J). 2004;80(5 Supl):S155-S162.

19. Weber F, Woolridge MW, Baum JD. An ultrasonographic study of the organisation of sucking and swallowing by newborn infants. Dev Med Child Neurol. 1986;28(1):19-24.

20. Cattoni DM, Neiva FCB, Zackiewicz DV, Andrade CRF. Fonoaudiologia e aleitamento materno: algumas contribuições. PróFono.1998;10(1):45-50.

21. Neiva FCB, Cattoni DM, Ramos JLA, Issler H. Desmame precoce: implicações para o desenvolvimento motor-oral. J Pediatr. 2003;79(1):712.

22. Hernandez AM. Atuação fonoaudiológica com recém-nascidos e lactentes disfágicos. In: Hernandez AM, Marchesan IQ. Atuação fonoaudiológica no ambiente hospitalar. Rio de Janeiro: Revinter; c2001. p.1-37.

23. Medoff-Cooper B, Verklan T, Carlson S. The development of sucking patterns and physiologic correlates in very-low-birth-weight infants. Nurs Res. 1993;42(2):100-5.

24. Silva RNM. Fatores que interferem na sucção/deglutição/respiração do prematuro. In: Lopes SMB, Lopes JMA. Follow up do recém-nascido de alto risco. Rio de Janeiro: Medsi; 1999. p. 275-300.

25. Glass RP, Wolf LS. A global perspective on feeding assessment in the neonatal intensive care unit. Am J Occup Ther. 1994;48(6):514-26.

26. Gamburgo LJL, Munhoz SRM, Amstalden LG. Alimentação do recém-nascido: aleitamento natural, mamadeira e copinho. Fono Atual. 2002;5(20):39-47.

27. Neiva FC, Leone C, Leone CR. Non-nutritive sucking scoring system for preterm newborns. Acta Paediatr. 2008;97(10):1370-5.

28. Neiva FCB, Leone CR. Sucção em recém-nascidos pré-termo e estimulação da sucção. Pró-Fono. 2006;18(2):141-50.

29. Morris SE, Klein MD. Pre-feeding skills: a comprehensive resourse for feeding development, therapy skill builders. Tucson, Arizona: Therapy Skill Builders; 1987.

30. Matias EL, Mello DFO. O seguimento fonoaudiológico e a participação materna na assistência ao recém-nascido pré-termo e de baixo peso. Rev Fonoaudiol Brasil. 2003;2(3):60-4. 